\title{
LINEAMIENTOS Y DIRECTRICES PARA LA TRANSFORMACIÓN Y PERFECCIONAMIENTO DE LA FORMACIÓN DE DOCENTES
}

Este artículo informa acerca de los principales asuntos tratados y de las conclusiones y proyecciones del encuentro nacional "Propuestas de Políticas sobre Formación Docente"1. Era la primera oportunidad en que se reunían, en un mismo espacio, representantes de todas las instituciones vinculadas con la formación inicial de docentes, con el compromiso de explorar y concordar formas de mejorarla, dado que reconocen en ésta el punto de partida para que niños y jóvenes puedan desarrollar a plenitud sus talentos en la escuela.

\section{Escenario actual}

Los datos presentados y la discusión posterior revelan que el país se encuentra en una etapa crucial y muy compleja de su reforma educacional, en la que la formación inicial y continua de los profesores es la variable prioritaria. Si bien ha habido avances en aspectos tales como el mejoramiento del currículo y la incorporación de recursos económicos para optimizar la alimentación, la creación de nuevos espacios, la adquisición de nuevos textos y computadores, y para permitir ejecutar una política de incentivos a los profesores de acuerdo con una evaluación previa, se hace evidente que ello por sí solo no es suficiente; lo que aparece como realmente relevante es, por cierto, el uso que hagan las comunidades escolares de estos aportes, en especial del trabajo docente.

1 Encuentro realizado en Santiago de Chile, entre el 6 y 7 de octubre de 2005, que contó con la participación de rectores y representantes de más de sesenta instituciones de educación superior formadoras de docentes, entre las que se contaban universidades públicas y privadas e institutos profesionales. El encuentro tuvo como base el Informe Preliminar de la Comisión sobre Formación Inicial Docente, presidida por el rector de la Universidad Metropolitana de Ciencias de la Educación, Raúl Navarro. 
Dado que hay un reconocimiento de que la labor principal se encuentra radicada en el profesor, se señaló la importancia de abordar las diversas dimensiones que inciden en su educación y posterior desarrollo profesional, es decir, formación inicial, perfeccionamiento continuo, condiciones de trabajo en escuelas y liceos, e institucionalidad ministerial para apoyarlos de manera adecuada.

La discusión se centró fundamentalmente en el tema de la formación inicial de los profesores, dado que representa la variable crucial para mejorar la calidad de la educación en esta etapa.

El intercambio de opiniones permitió destacar algunos avances: en primer lugar, la recuperación del sitial, dignidad y trascendencia de la educación en nuestra sociedad. La transformación educacional de los últimos 16 años ha significado el mayor esfuerzo de nuestra historia, valorado por la inmensa mayoría de las familias chilenas, para quienes la educación representa actualmente la esperanza de una mejor calidad de vida. Tal como ocurre en el resto del mundo, esa expectativa está generando presiones en los sistemas educativos, los que requieren ajustar sus modelos con vistas a preparar a las nuevas generaciones para la sociedad del conocimiento.

Un segundo avance importante, que se puso también de relieve, es la mejora sustancial de las condiciones para el ejercicio de la docencia. Se ha visto un aumento progresivo, tanto en las remuneraciones de los profesores como en las posibilidades de perfeccionamiento; se cuenta con un Estatuto Docente que, aunque con imperfecciones, ha significado un paso importante en la regulación del trabajo y la profesionalización de los educadores. El país dispone de un sistema de evaluación docente, de carácter formativo, que permite a éstos conocer sus fortalezas y debilidades y, según ese diagnóstico, mejorar su desempeño en el aula. Se han establecido incentivos monetarios para los profesores destacados, como la Asignación de Excelencia Pedagógica o la Asignación Variable por Desempeño Individual, creada a partir de la instalación 
del proceso de evaluación docente. Se ha puesto en práctica, por ley — aprobada este año-, la concursabilidad del cargo de director, hecho que representa una oportunidad de ascenso para los profesores que cuentan con competencias directivas y de liderazgo.

En tercer lugar, se destacó que, ya que hoy la educación es mejor valorada por la sociedad y los profesores trabajan en condiciones dignas, hay un creciente interés por parte de los buenos alumnos por estudiar pedagogía: en los últimos diez años, la matrícula de las carreras de educación aumentó de 25 mil a 67 mil alumnos; por otra parte, el puntaje promedio de los postulantes a las mismas experimentó un alza de 536 a 624 puntos. Además, la postulación a carreras del área pedagógica aumentó como la primera opción entre quienes desean acceder a la educación superior. A ello ha contribuido enormemente la decisión política de crear, en 1998, la Beca para Estudiantes Destacados de Pedagogía, beneficiando desde entonces a 1.853 postulantes que han ponderado más de 600 puntos en la prueba de ingreso a las universidades chilenas.

En cuarto lugar, los diversos asistentes al encuentro mencionaron la importancia del cada vez más potente vínculo entre la formación de profesores y la práctica docente en el aula. Este fue uno de los méritos del Programa de Fortalecimiento de la Formación Inicial, que destinó recursos, entre 1997 y 2002, para diversos propósitos, entre los cuales la reforma curricular de los programas de pedagogía de 17 universidades públicas y privadas (que acentuaba el nexo con la escuela como eje articulador de la formación docente) fue el más importante.

Por otra parte, se mencionó que la renovación de los cuerpos académicos de las carreras de pedagogía, una de las necesidades demandadas en el Informe Preliminar de la Comisión sobre Formación Inicial, es posible hoy en las 16 universidades estatales. De acuerdo con la ley aprobada este año, que les otorgó facultades financieras, estos planteles podrán asignar un bono especial de un 
mes por año de servicio, con tope de once meses, a los funcionarios a contrata o de carrera, cuya edad supere los 65 años (en el caso de los hombres) y los 60 años (en el caso de las mujeres), y que comuniquen su decisión de renunciar en forma voluntaria.

Se informó que en 15 universidades del Consejo de Rectores ya se está llevando a cabo un rediseño curricular de las pedagogías con aportes del Programa de Mejoramiento de la Calidad y Equidad de la Educación Superior (MECESUP), iniciativa a la que se han sumado, a partir de este año, cuatro proyectos MECESUP, impulsados por consorcios de universidades, con el propósito de diseñar carreras de Pedagogía Básica con mención en Lenguaje, Matemáticas, Ciencias Naturales e Historia y Ciencias Sociales. Además, en colaboración con las universidades de Chile y de Concepción, se han definido estándares para la formación de profesores de enseñanza media en Matemáticas, Biología, Física y Química.

Un último hecho relevante es el aumento del interés de las instituciones de educación superior por acreditar sus carreras de pedagogía, práctica que sigue siendo voluntaria en tanto se aprueba la ley. A la fecha, de 508 carreras de pedagogía —incluidos 23 programas especiales que imparten 65 instituciones-, la Comisión Nacional de Acreditación de Pregrado (CNAP) ha acreditado 73 carreras, por periodos que van de dos a seis años.

\section{Proyecciones y tareas para el periodo 2006-2010}

Se puede afirmar que, junto con el respaldo social, hay señales claras de una decisión política, lo que ha permitido lograr parte de los avances que se han reseñado anteriormente. Como resulta obvio en un proceso tan profundo de reforma educacional, aún existen múltiples tareas necesarias para mejorar la formación inicial de los profesores.

Ahora bien, ¿cuáles son las principales metas a las que se deben apuntar de manera conjunta? 
1. Definición de una política integral sobre formación inicial y permanente del profesorado.

2. Acreditación obligatoria de las carreras de pedagogía. Se espera que el Congreso apruebe el proyecto de ley a fines del presente año, lo que permitirá fortalecer los mecanismos de regulación y autorregulación de las instituciones de educación superior, para asegurar una formación de calidad en esta área.

3. Definición apropiada de un perfil de egreso del profesor, que deberá considerar las competencias pedagógicas mínimas necesarias, lo específico de cada especialidad y el grado académico ofrecido. En el encuentro se recomendó que, en este perfil, se incorpore el otorgamiento del grado de licenciado.

4. Priorización de lo que es central en la formación de profesores: la definición del saber específico de esta profesión, cuál es, cómo los seres humanos aprenden. Se requiere, además, que exista una adecuada articulación entre este saber pedagógico y el saber disciplinario. Los profesores deben ser expertos en aprendizaje. Actualmente, se avanza hacia un cambio de paradigma de "enseñante" a "facilitador del aprendizaje"; en efecto, cada día se usa más el modelo médico para la enseñanza en la educación (estudio de casos, investigación docente, evaluación de desempeño, entre otras estrategias).

5. Establecimiento de cambios curriculares en las carreras pedagógicas. Algunas propuestas fueron:

- Lograr una vinculación más estrecha entre la formación universitaria y la práctica en establecimientos educacionales, de modo de tener una formación de mayor contacto con el aula. Es indudable que, en los últimos tiempos, ha habido avances en este sentido, pero aún no se concibe en todas las instituciones formadoras a la escuela como una instancia importante para la formación de los docentes, como lo son, en otra área, los hospitales para los estudiantes de medicina, por ejemplo.

- Buscar algunos mecanismos para que un mayor número de profesores de aula, del sistema escolar, puedan participar 
en el proceso de formación de los estudiantes de pedagogía en universidades e institutos profesionales.

- Establecer una mayor vinculación entre las escuelas de pedagogía y las facultades o escuelas que imparten las disciplinas al interior de la misma universidad, puesto que los datos indican que este trabajo conjunto es débil.

- Fortalecer la relación entre la formación inicial y el currículo escolar, particularmente en función de los logros esperados en el alumno (estándares de aprendizaje); para ello se está trabajando en el diseño de mapas de progreso para cada asignatura y nivel.

- Fomentar la especialización obligatoria para profesores de enseñanza básica del segundo ciclo y ofrecer una mención en el primer ciclo. Hoy, sólo el $20 \%$ de los estudiantes de pedagogía básica del país estudia carreras que incorporan una especialización.

- Definir estándares para la formación de docentes y evaluar a los estudiantes que egresan conforme a dichos estándares.

6. Diseño de una política de inducción de los recién egresados en el sistema escolar, de manera de acompañarlos en una primera etapa de inserción en la docencia, alentando el desarrollo de nuevas prácticas docentes. En ello ha trabajado una comisión especial que próximamente entregará una propuesta.

7. En la misma línea, creación y aumento, según sea el caso, de las posibilidades de impartir formación pedagógica a licenciados en distintas disciplinas, de modo de aumentar el número de profesores con especialización.

8. Cierre de programas virtuales de formación docente y otros de baja exigencia académica y con escasas horas presenciales. Una carrera de pedagogía debe durar al menos cuatro años, tener elevadas exigencias académicas y recibir a quienes obtienen puntaje de mérito en la Prueba de Selección Universitaria (PSU). El ministro de Educación, tras hacer un llamado a las instituciones de educación superior al respecto, celebró que este desafío ya hubiera sido asumido por varias de ellas y expresó su confianza en que su ejemplo se replicará en otras del país. 
9. Incremento del número de becas para los mejores estudiantes de pedagogía. El país no puede proyectarse en términos de innovación, ciencia y tecnología si no se estimula la formación de profesores en áreas deficitarias (matemáticas, ciencias e inglés). Por ello, parte de los recursos que genere la ley que estableció un royalty a la producción de cobre —en virtud de la cual se creará un Fondo para la Innovación- se destinará a incrementar el número de becas para estudiantes de pedagogía en estas asignaturas.

10. Estudio de una propuesta de aplicación de un examen, al momento de egresar de los programas académicos de pedagogía, como requisito para ejercer en establecimientos subvencionados por el Estado.

11. Superación de las fallas de coordinación entre las unidades académicas de que depende cada carrera --disciplinarias y pedagógicas-, ya que ello genera problemas no sólo de coherencia e integralidad en la formación que se entrega $-y$ de pertinencia del enfoque de los contenidos-, sino también de gestión de la carrera, falta de vinculación entre propósitos y proyectos, dificultad en la identificación de los alumnos con su calidad de futuro profesor, descoordinación de los cursos del plan curricular, escaso trabajo conjunto de planificación y evaluación de la carrera, entre otros.

12. Realización de un análisis, en las diversas escuelas y facultades de Educación, de los resultados del estudio: "Caracterización de las carreras de pedagogía a partir de estudios de carreras acreditadas por la CNAP", con el propósito de perfeccionar los aspectos señalados como deficitarios.

13. Estudio, por parte del Colegio de Profesores, de otorgar mayores facultades al Ministerio de Educación para la regulación del sistema de enseñanza superior, especialmente en lo concerniente a las carreras de pedagogía.

14. Diálogo permanente entre estudiantes y académicos, que redundará en el perfeccionamiento de los planes curriculares, el cual deberá considerar la importancia de la formación general 
y de la inmersión temprana, puesto que la reflexión sobre la práctica profesional es vital para el aprendizaje. Los estudiantes demandan docentes consecuentes, que encarnen valores, que sean referentes idóneos para su ejercicio profesional y que estén llanos a integrar los contenidos emergentes en la formación de profesionales de la educación.

15. Reforzamiento de la investigación en educación. La escasa vinculación de los formadores con los problemas y desafíos educativos del país acentúa la brecha entre universidades y escuelas de pedagogía. Afianzar la investigación educativa requiere más recursos, por ello se anunció la asignación de 309 millones de pesos para investigación educativa en la Ley de Presupuesto 2006. Este monto se destinará a:

- Desarrollar un sistema más articulado de bases de datos.

- Crear un fondo de investigación educativa, con participación de expertos.

- Elaborar estudios de seguimiento de actores del sistema educativo a lo largo de su trayectoria (longitudinales o de panel).

Finalmente, los representantes reunidos, más el Colegio de Profesores y el MINEDUC, acordaron una agenda de trabajo y un compromiso por la calidad de la formación inicial docente, con lo cual se espera implementar y desarrollar un seguimiento de las pautas referidas anteriormente. 